\title{
The role of topic vaginal products in posthysterectomy vaginal atrophy
}

\author{
Nicolae Bacalbasa ${ }^{1,2}$, Irina Balescu ${ }^{3}$, Adnan Ad Aloul|, ${ }^{4,5}$, Roxana Bohiltea ${ }^{1,6}$, Bogdan Socea ${ }^{7,8}$, \\ Bogdan Ursut ${ }^{1,9}$, Alexandru Filipescu ${ }^{1,10}$ \\ ${ }^{1}$ Department of Obstetrics and Gynecology, "Carol Davila" University of Medicine and Pharmacy, Bucharest, Romania \\ ${ }^{2}$ Department of Visceral Surgery, Center of Excellence in Translational Medicine, \\ Fundeni Clinical Institute, Bucharest, Romania \\ ${ }^{3}$ Department of Visceral surgery, Ponderas Academic Hospital, Bucharest, Romania \\ ${ }^{4}$ Department of Surgery, Ramnicu Sarat County Hospital, Buzau, Romania \\ ${ }^{5}$ Department of Surgery, "Titu Maiorescu" University, Bucharest, Romania \\ ${ }^{6}$ Department of Obstetrics and Gynecology, University Emergency Hospital, Bucharest, Romania \\ ${ }^{7}$ Department of Surgery, "Carol Davila" University of Medicine and Pharmacy, Bucharest, Romania \\ ${ }^{8}$ Department of Surgery, "Sf. Pantelimon" Clinical Emergency Hospital, Bucharest, Romania \\ ${ }^{9}$ Department of Surgery, "Agrippa Ionescu" Clinical Emergency Hospital, Bucharest, Romania \\ ${ }^{10}$ Department of Obstetrics and Gynecology, Elias Emergency Hospital, Bucharest, Romania
}

\begin{abstract}
Vaginal atrophy represents a frequently encountered condition which is significantly correlated with decreased amounts of circulating oestrogen levels. It is usually characterised by the presence of burning, itching, irritation and dyspareunia. In such cases attention was focused on identifying a topic product which might alleviate these symptoms and stop the deterioration process of the vaginal lining. One product which seems to have an effective role in order to stop this process and to offer a good local control of the disease should contain anti-inflammatory, healing and antiseptic principles. The aim of the current paper is to study the possible benefits of Cerviron a topic product with intravaginal administration which contains seven active principles in order to offer the above mentioned effects.
\end{abstract}

Keywords: vaginal atrophy, oestrogen, menopause, Cerviron

\section{INTRODUCTION}

Although attention has been widely focused worldwide on performing conservative procedures in order to preserve future fertility and to avoid pelvic disorders, hysterectomy still represents the cornerstone gynaecological procedure worldwide $(1,2)$. Meanwhile, although surgical procedure significantly improved and minimally invasive surgery has been widely implemented in gynaecology surgery leading to a faster recovery, a lower degree of pain and a faster return to normal social life $(3,4)$, it is still associated with significant psychological impact (5-8). A significant factor which might influence the postoperative psychological status of the patient is represented by the indication ewhich leaded to the necessity of performing a hysterectomy; therefore while patients submitted to surgery for benign conditions will probably have a feeling of relief after surgery (due to the fact that their problem is basically solved), patients submitted to surgery for malignant conditions will still report a feeling of anxiety and depression due to the diagnostic of cancer (9). 


\section{POSTMENOPAUSAL OESTROGEN DEFICIENCY}

Meanwhile, another significant modification which appears after hysterectomy is related to hormonal modifications such as oestrogen decrease. This hormonal imbalance seems to be responsive for a significant number of vascular, urogenital and musculoskeletal changes which have a psychological impact on the patients' state and on their quality of life $(10,11)$. One of the most common complaints which are described to develop in close connection with hysterectomy and estrogen level drop down is represented by vaginal atrophy. This condition is usually associated with symptoms like vaginal dryness - reported in up to half of patients, pruritus, itching, burning, dyspareunia (12). In order to better control these symptoms the first and most appropriate solution which was imagined consisted of local administration of oestrogen related topics (13). This way of administration offers an effective local absorption of this active principle and improves the local symptoms; meanwhile, although the systemic absorption is limited, it is still present and might have a serious impact on the risk of recurrence in patients with previous history of oestrogen dependent malignancies such as breast, endometrial or ovarian cancer (14-18).

\section{VAGINAL ATROPHY (ATROPHIC VAGINITIS)}

As mentioned before, one of the most commonly encountered modifications reported after total hysterectomy with bilateral adnexectomy is represented by vaginal atrophy. Also known as atrophic vaginitis, this condition is considered to be an unspecific vaginitis, characterised by the presence of a diminished elasticity of the vaginal mucosa, vaginal dryness and a thinner vaginal lining. Meanwhile, other symptoms such as reddish aspect of the vulvo-vaginal area or purulent discharge have been reported; in such cases a differential diagnostic with infectious vaginitis is needed in order to establish the right origin of the affection and to correctly conduct the treatment. In such cases direct visualisation provides the identification an extremely thin vaginal lining will establish the right diagnostic $(19,20)$. In the meantime, association of imagistic studies such as transvaginal and even abdominal ultrasound provides a more objective information - the exact dimension of the vaginal mucosa; according to this information the degree of atrophy can be precisely determined and a proper treatment can be applied (21).

\section{THERAPEUTIC STRATEGIES IN VAGINAL ATROPHY}

In order to alleviate the above mentioned symptoms without increasing the risks of exposure to a high amount of unopposed oestrogen, different topical products have been proposed with various results. Therefore, in such cases a proper topic product should alleviate the symptoms, should decrease the risks of apparition of microtraumatisms and secondary infections and should provide an effective healing process.

In this respect, non-hormonal moisturizing vaginal gels or ovules have been widely investigated so far. Interesting results have been reported after the three times per week use of a gel consisting of purified water, glycerine, hydrogenated palm oil glyceride, polycarbophil and sorbic acid; surprisingly such products reported a superior efficacy when compared to oestrogen based products (22). Other effective agents are represented by homeopathic agents such as bryonia, belladonna, lycopodium, Calendula officinalis, Curcuma longa, Hydrastis canadensis or Thymus vulgaris or vitamins such as vitamin D, E or alpha-tocopherol (23-27). Another active principle which has been proposed is the one represented by $\mathrm{pH}$ balanced vaginal gel, which seems to re-establish the normal environmental vaginal $\mathrm{pH}$, and to create a proper medium for an adequate development of a normal vaginal flora; the main active principle in such cases is represented by the lactic acid which provides a $\mathrm{pH}$ value of 4.2 (28).

An interesting product which includes multiple active principles such as homeopathic agents - $\mathrm{Ca}$ lendula officinalis, Curcuma longa, Hydrastis canadensis or Thymus vulgaris, collagen, hexylresorcinol and bismuth subgallate is represented by Cerviron, a topic agent with intravaginal administration; in atrophic vaginitis is recommended that this product should be administrated 10 days to three weeks in order to reduce local inflammation, to diminish the risk of infection and to provide an efficient healing process of the vaginal mucosa. This product also seem to increase the efficacy of system antibiotic treatment due to the association of hexylresorcinol and to minimise the risk of infectious complications. Meanwhile, the presence of bismuth subgallate seems to diminish the risk of bleeding and to have a hemostatic effect while collagen provides a protective pellicle at the level of the vaginal lining (29-32).

\section{CONCLUSIONS}

Although surgical technique significantly improved and so did the postoperative outcome of patients submitted to total hysterectomy with bilateral adnexectomy for both benign and malignant conditions, certain postoperative sequels such as vaginal atrophy still have a significant impact on the quality of life. It has been widely demonstrated that low 
levels of oestrogens represent the most important factor for the apparition of these modifications and therefore, topic oestrogen products have been imagined. However, we should not omit the fact that although reduced, systemic absorption of oestrogen might occur, inducing significant risks especially in cases with previous history of oestrogen related malignancies. In this respect, attention was focused on determining which are the active principles which should be introduced in the composition of topical agents in order to diminish and alleviate these symptoms. A promising product which seem to associate multiple active principles is represented by Cerviron, a product with topic, intravaginal administration which associates both pharmacological and homeopathic agents but which does not contain at all any pure or oestrogen derivate.

\section{Conflict of interest: none declared Financial support: none declared}

\section{REFERENCES}

1. Chen I, Wise MR, Dunn S, Anderson G, Degani N, Lefebvre G, Bierman AS. Social and geographic determinants of hysterectomy in Ontario: a population-based retrospective cross-sectional analysis. J Obstet Gynaecol Can. 2017;39:861-869.

2. Canadian Institute for Health Information (ClHI). Inpatient hospitalizations, surgeries, newborns, and childbirth indicators, 2016-2017. Ottawa: ClHI; 2018. Available at: https://secure.cihi.ca/ estore/productFamily.htm? pf=PFC3714\&lang=en\&media=0.

3. Perron-Burdick M, Yamamoto M, Zaritsky E. Same-day discharge after laparoscopic hysterectomy. Obstet Gynecol. 2011;117:1136-1141.

4. Jennings AJ, Spencer RJ, Medlin E, Rice LW, Uppal S. Predictors of 30-day readmission and impact of same-day discharge in laparoscopic hysterectomy. Am J Obstet Gynecol. 2015;213:344.e1-7.

5. Ewalds-Kvist SB, Hirvonen T, Kvist M, Lertola K, Niemela P. Depression, anxiety, hostility and hysterectomy. J Psychosom Obstet Gynaecol. 2005;26:193-204.

6. Costantini E, Porena M, Lazzeri M, Mearini L, Bini V, Zucchi A. Changes in female sexual function after pelvic organ prolapse repair: role of hysterectomy. Int Urogynecol J. 2013;24:1481-1487.

7. Lowenstein L, Yarnitsky D, Gruenwald I, Deutsch M, Sprecher E, Gedalia $\mathrm{U}$, Vardi Y. Does hysterectomy affect genital sensation? Eur J Obstet Gynecol Reprod Biol. 2005;119:242-245.

8. Aziz A, Bergquist C, Nordholm L, Moller A, Silfverstolpe G. Prophylactic oophorectomy at elective hysterectomy: effects on psychological well-being at 1-year follow-up and its correlations to sexuality. Maturitas. 2005;51:349-57.

9. Cooper R, Mishra G, Hardy R, Kuh D. Hysterectomy and subsequent psychological health: Findings from a British birth cohort study. Journal of Affective Disorders. 2009;115:122-130.

10. The North American Menopause Society. The role of local vaginal estrogen for treatment of vaginal atrophy in postmenopausal women: 2007 position statement of The North American Menopause Society. Menopause. 2007;14:355-369.

11. Archer DF. Efficacy and tolerability of local estrogen therapy for urogenital atrophy. Menopause. 2010;17(1):194-203.

12. Bachmann GA, Nevadunsky NS. Diagnosis and treatment of atrophic vaginitis. American Family Physician. 2000;61:3090-6.

13. Cardozo L, Bachmann G, McClish D, Fonda D, Birgerson L. Metaanalysis of estrogen therapy in the management of urogenital atrophy in postmenopausal women: second report of the Hormones and Urogenital Therapy Committee. Obstetrics and Gynecology. 1998;92:722-727.

14. Ballagh SA. Vaginal hormone therapy for urogenital and menopausal symptoms. Seminars in Reproductive Medicine. 2005;23:126-140.

15. Rioux JE, Devlin MC, Gelfand MM, Steinberg WM, Heppburn DS. 17-Estradiol vaginal tablets versus conjugated equine estrogen cream to relieve menopausal atrophic vaginitis. Menopause. 2000;7:156-161.

16. Suckling JA, Kennedy R, Lethaby A, Roberts H. Local oestrogen therapy for vaginal atrophy in post menopausal women. Cochrane Database of Systematic Reviews. 2006;4:CD001500.

17. Bachmann G, Bouchard C, Hoppe D, Ranganath R, Altomare C, Vieweg A, Graepel J, Helzner E. Efficacy and safety of low-dose regimens of conjugated estrogens cream administered vaginally. Menopause. 2009;16:719-727.

18. Sturdee DW, Panay N, on behalf of the International Menopause Society Writing Group. Recommendations for the management of postmenopausal vaginal atrophy. Climacteric. 2010;13:509-522.

19. Nappi RE, Kokot-Kierepa M. Vaginal health: Insights, Views \& Attitudes (VIVA) - Results from an international survey. Climacteric. 2012; 15:36-44.

20. Nappi RE, Palacios S. Impact of vulvovaginal atrophy on sexual health and quality of life at postmenopause. Climacteric J Int Menopause Soc. 2014;17:3-9.

21. Balica A, Wald-Spielman D, Schertz K, Egan S, Bachmann G. Assessing the thickness of the vaginal wall and vaginal mucosa in premenopausal versus post-menopausal women by transabdominal ultrasound: A feasibility study. Maturitas. 2107;102:69-72.

22. Bygdeman M, Swahn ML. Replens versus dienoestriol cream in the symptomatic treatment of vaginal atrophy in postmenopausal women. Maturitas. 1996;23(3):259-63.

23. Weed S. Menopausal Years: the wise woman way - alternative approaches for women, vol. 30-90. Woodstock, New York: Ash Tree; 1992.

24. Ronningen $\mathrm{H}$, Langeland $\mathrm{N}$. Indomethacin treatment in osteoarthritis of the hip joint. Acta Orthop Scand. 1979;50:169-174.

25. Yildirim B, Kaleli B, Duzcan E, Topuz O. The effects of postmenopausal Vitamin D treatment on vaginal atrophy. Maturitas. 2004;49:334-337.

26. Giardinelli M. Effect of alpha-tocopherol in some disorders of the menopause and in atrophy of the vaginal mucosa. Minerva Ginecol. 1952;4:579-587.

27. Johnston SL, Farrell SA, Bouchard C, Farrell SA, Beckerson LA, Comeau M, Johnston SL, Lefebvre G, Papaioannou A; SOGC Joint CommitteeClinical Practice Gynaecology and Urogynaecology. Detection and management of urogenital atrophy. J Obstet Gynaecol Can. 2004;26:503-515.

28. Lee Y-K, Chung HH, Kim JW, Park N-H, Song YS, Kang SB. Vaginal $\mathrm{pH}$-balanced gel for the control of atrophic vaginitis among breast cancer survivors. Obstetrics and Gynecology. 2011;117(4):922-927.

29. Nicolaus C, Junghanns S, Hartmann A, Murillo R, Ganzera M, Merfort I. In vitro studies to evaluate the wound healing properties of Calendula officinalis extracts. J Ethnopharmacol. 2017 Jan 20;196:94-103.

30. Kaneda Y, Torii M, Tanaka T, Aikawa M. In vitro effects of berberine lamblia and Trichomonas vaginalis. Ann Trop Med Parasitol. 1991 Aug;85(4):417-25.

31. Patil SM, Ramu R, Shirahatti PS, Shivamallu C, Amachawadi RG. A systematic review on ethnopharmacology, ph ytochemistry and pharmacological aspects of Thymus vulgaris Linn. Heliyon. 2021 May 1;7(5):e07054.

32. Bolke L, Schlippe G, Gerß J, Voss W. A Collagen Supplement Improves Skin Hydration, Elasticity, Roughness, and Density: Results of a Randomized, Placebo-Controlled, Blind Study. Nutrients. 2019 Oct 17;11(10):2494. sulphate on the growth and structure of Entamoeba histolytica, Giardia 